\title{
The coupling of capillary electrophoresis to ICP-MS
}

\author{
B. Michalke and P. Schramel
}

GSF - National Research Center for Environment and Health, Institute for Ecological Chemistry, Ingolstädter Landstr. 1, 85764 Neuherberg, Germany

Improvements in coupling capillary electrophoresis (CE) to inductively coupled plasma mass spectrometry (ICP-MS) are discussed for speciation of Pt-, As- and Se compounds. They are due to the use of long capillaries and to the separation of the CE separation step (focusing) from the ICP-MS detection step (mobilisation).

apillary electrophoresis has become a matter of interest in element speciation predominantly owing to its high separation potential, fast separation capabilities, and the lack of interactions between the analyte species and the stationary phase [1]. The latter may induce species alterations that have widely been described [2-5]. For several years coupling of $\mathrm{CE}$ to element selective detectors, such as ICP-MS, has been performed by few scientific groups with different success [6-10,11]. Most of these groups have used laboratory made CE-setups, installed at the sample introduction system of an ICP-MS. $30-50 \mathrm{~cm}$ capillaries of $50 \mathrm{~mm}$ i.d. have usually been used. Analyte species move towards an ICP-MS which acts as a detector. Species with the opposite charge migrate immediately into the inlet buffer vial and therefore are not monitored. In our investigations a commercial system, equipped with very long capillaries and a laboratory made interface $[8-10,12]$ were used. The whole system was operated in a special mode: the separation step was separated from the pressure driven detection step. This operational mode offered several advantages in comparison with conventional hyphenation techniques, such as:

(i) The absence of a suction flow. Liquid flow through the capillary, produced by a suction from the nebulisation gas stream would be very undesirable during the separation. The performance of CE separations would decrease drastically. This is already described in literature [6,13] when short capillaries were used, but was not seen in our former investigations with long capillaries having a total length of $150 \mathrm{~cm}[8-10,12]$. This is due to the flow resistance caused by the inner diameter and total length of the capillary and is defined by the law of Hagen-Poiseuille [14]:

$$
\frac{V}{t}=\frac{r^{4} \times \Pi \times(p-p)}{8 \times n \times L}
$$

$V=$ volume passing through the capillary per time " $t$ ", $r=$ radius (half diameter) of the capillary, $p 1$ and $p 2$ - pressures at capillary inlet and outlet, respectively, $n=$ viscosity of the buffer, $L=$ total length of the capillary.

The difference of $p 1-p 2$ is the suction force applied by nebulisation gas stream and $V / t$ is the suction flow. $V / t$ decreases, when $L$ increases. Hence, the long capillary together with the special design of the interface can reduce that suction flow to an insignificant value. Separations will not be interfered by a rapid liquid stream as mentioned in [6].

(ii) Time reduction. The coupling of a commercial $\mathrm{CE}$ device with ICP-MS typically needs long capillaries as the $\mathrm{CE}$ can rarely be positioned closely to the ICP-MS sample introduction system. The use of long capillaries and the lack of a suction driven flow results in long migration times. Thus, one big advantage of CE separations is lost. To overcome this problem the separation of the separation step from the detection step is helpful. Typically after $10-15 \min$ a sufficient separation is achieved. Then, the pressure driven detection step is started. Metal species are transported to ICP-MS within ca. $130 \mathrm{~s}$. This two step procedure is derived from capillary isoelectric focussing, where it is well established [15-17]. These separated molecule bands do not move to the detector until a pressure is applied at the capillary inlet. Then, the bands move one after the other to the outlet. The pattern of separated molecules is not impaired and is monitored without interferences at the end of capillary very quickly. Short analysis times are principally required when investigating labile species which show rapid degradations or transformations [9].

(iii) Improvement of nebulisation. The pressure driven detection step results in a flow of ca. $1.5 \mu \mathrm{L} \mathrm{min}{ }^{-1}$. The molecular bands are forced to move at $1.5 \mathrm{~cm} \mathrm{~s}^{-1}\left(90 \mathrm{~cm} \mathrm{~min}^{-1}\right)$. 
This liquid stream increases the efficiency of nebulisation. The optimum value was around $1-2 \mu \mathrm{L} / \mathrm{min}$ (compared to a flow during separation of $10 \mu \mathrm{L} / \mathrm{h}$ ). Furthermore, this flow provides a nearly quantitative transport to ICP-MS; in case of a low retiring velocity of molecules from the capillary (e.g. during separation mode), charged molecules try to migrate towards the Pt-electrode in counter direction to the very slow $(10 \mu \mathrm{L} / \mathrm{h})$ sheath flow. This results in a loss of sensitivity, as these species are no more nebulised or transported to ICP-MS. Besides, the peak area is a constant value for a given detector sensitivity/instrumentation and analyte concentration. It is further a function of peak height and the residence time of the analyte in the detector. Therefore, peak height increases due to a constant peak area, when reducing the residence time by accelerating the analyte flow [8].

(iv) Improvement of concentration detection limit (DL). The use of long capillaries and the differentiation of separation and detection is a useful prerequisite for improving concentration detection limits. Long capillaries provide sufficient space for an extended "buffer sandwich" consisting of a large sample plug and of adequate large amounts of stacking (leading) and terminating electrolytes.

Stacking electrolytes are necessary to achieve still good separation although the sample volume is quite large. Their amount is typically in the same volume range as the sample itself. The sample volume can be increased from typically $5 \mathrm{~nL}$ to ca. $300-400 \mathrm{~nL}$ without any significant loss in resolution. According to the sample volume increase also the concentration detection limit is improved. But this "sandwich" of buffers and sample needs a considerable capillary length, the more, the sample volume is increased. For example, a sandwich consisting of $300 \mathrm{~nL}$ sample $+300 \mathrm{~nL}$ leading electrolyte needs about $30 \mathrm{~cm}$ of capillary. Additional capillary length is necessary for ITP/pH stacking and the CZE separation process for sufficient resolution. Although the stacking precedes the CZE separation, the total "separation step" usually can be stopped after $10-15$ min by starting the pressure driven detection step.

(v) Improvement of separation. CZE separation is based on the differences of charge-to-mass ratios. When this difference between two species is low also the resolution is low. One solution for overcoming this problem is a $\mathrm{pH}$ stacking, which reverses the mobility of one of the species according to (only small) differences of their $\mathrm{pK}_{\mathrm{A}}$ values. Using conventional techniques the backward moving species reaches the inlet buffer vial and thus cannot be monitored at ICP-MS. Therefore, this improvement in separation cannot be used with conventional techniques, except when a very fast EOF is directed towards ICP-MS. However, a fast EOF possibly interferes with resolution efficiency and its existance principally is limited to more alcaline conditions. The same situation is seen when analysing cations and anions in one run. Only a (very) fast EOF helps to detect both cations and anions in one run using the conventional technique.

However, when a sample/buffer sandwich is positioned in the middle of a long capillary, one species migrates towards ICP-MS, while the other one towards the inlet buffer vial. The separation ends by starting the pressure driven detection step before the species leave the capillary (either at inlet or outlet). Both species (or both, anions and cations) are then monitored clearly resolved by ICP-MS. (vi) Application of a highly resolving technique: cIEF. Capillary isoelectric focusing is known for its superior resolution. This technique consists of a focusing step and a mobilisation step, which is analogous to the pressure driven detection step. Actually, the pressure driven detection step is a fundamental prerequisite for this method. The division of the two processes was the basic idea for differentiation of separation and detection also in CZE. In addition to the high resoltion provided by cIEF, also concentration detection limits are improved. Here, the total capillary is filled with sample (in $2 \%$ ampholytes). Therefore, the length of the capillary influences directly the concentration detection limit. With a $1.5 \mathrm{~m}$ capillary the sample volume is ca. $3 \mu \mathrm{L}$ in maximum, which is an increase by a factor of 590 compared to conventional sample volumes $(5 \mathrm{~nL})$. Furthermore, the resolution of cIEF is correlated to the $\mathrm{pH}$ gradient along the total length of the capillary. Thus, increased capillary length also increases the resolution.

This contribution focusses on the experiments demonstrating these advantages and discusses probably occurring problems. The described experiments were performed along the progress of developments for the CE-ICP-MS coupling and application to different element species.

\section{Experimental results}

Table I shows an overview of the experimental parameters used in this works.

\section{Time reduction, improvement of nebulisation and of concentration DL}

Figure 1 shows three electropherograms of $\mathrm{Na}_{2} \mathrm{PtCl}_{6} \times$ $6 \mathrm{H}_{2} \mathrm{O}$. Figure $1 \mathrm{~A}$ demonstrates a conventional operation without the division of separation from detection. The Ptspecies appears at 28.5 min, showing a peak width at halfheight of $50 \mathrm{~s}$. The peak height was 560 ions/s, the noise around 20 ions/s, resulting in a signal to noise ratio of 28 . The detection limit was calculated around $5 \mathrm{mg} / \mathrm{L}$. Figure 1B shows the same sample. But here, the detection step was started after 10 min of separation and the Pt-species moved to ICP-MS within $18 \mathrm{~s}$. Thus, the total analysis time was now $10 \mathrm{~min}+18 \mathrm{~s}$, which means a 3 -fold reduction in time. Also, the nebulisation efficiency was improved. This is seen by the peak height reaching ca. 58000 ions/s (noise 230 ions/s). The signal to noise ratio thus is 250 and the calculated detection limit is $500 \mathrm{mg} / \mathrm{L}$. The peak width at halfheight was determined at $1.5 \mathrm{~s}$.

Figure $1 \mathrm{C}$, however, shows the same sample additionally using a stacking procedure. Again, the detection step was started after $10 \mathrm{~min}$ of separation and the Pt-species moved to ICP-MS within $18 \mathrm{~s}$. The total analysis time was $10 \mathrm{~min}$ $+18 \mathrm{~s}$, too. The large sample volume and the additional application of sample stacking improved the concentration DL considerably without a significant loss in resolution by peak broadening. The signal to noise ratio here is 86 (but for a concentration of $100 \mu \mathrm{g} / \mathrm{L}$, not $100 \mathrm{mg} / \mathrm{L}$ !) and the calculated detection limit is $1 \mu \mathrm{g} / \mathrm{L}$. The peak width at halfheight was determined at $1.85 \mathrm{~s}$. 


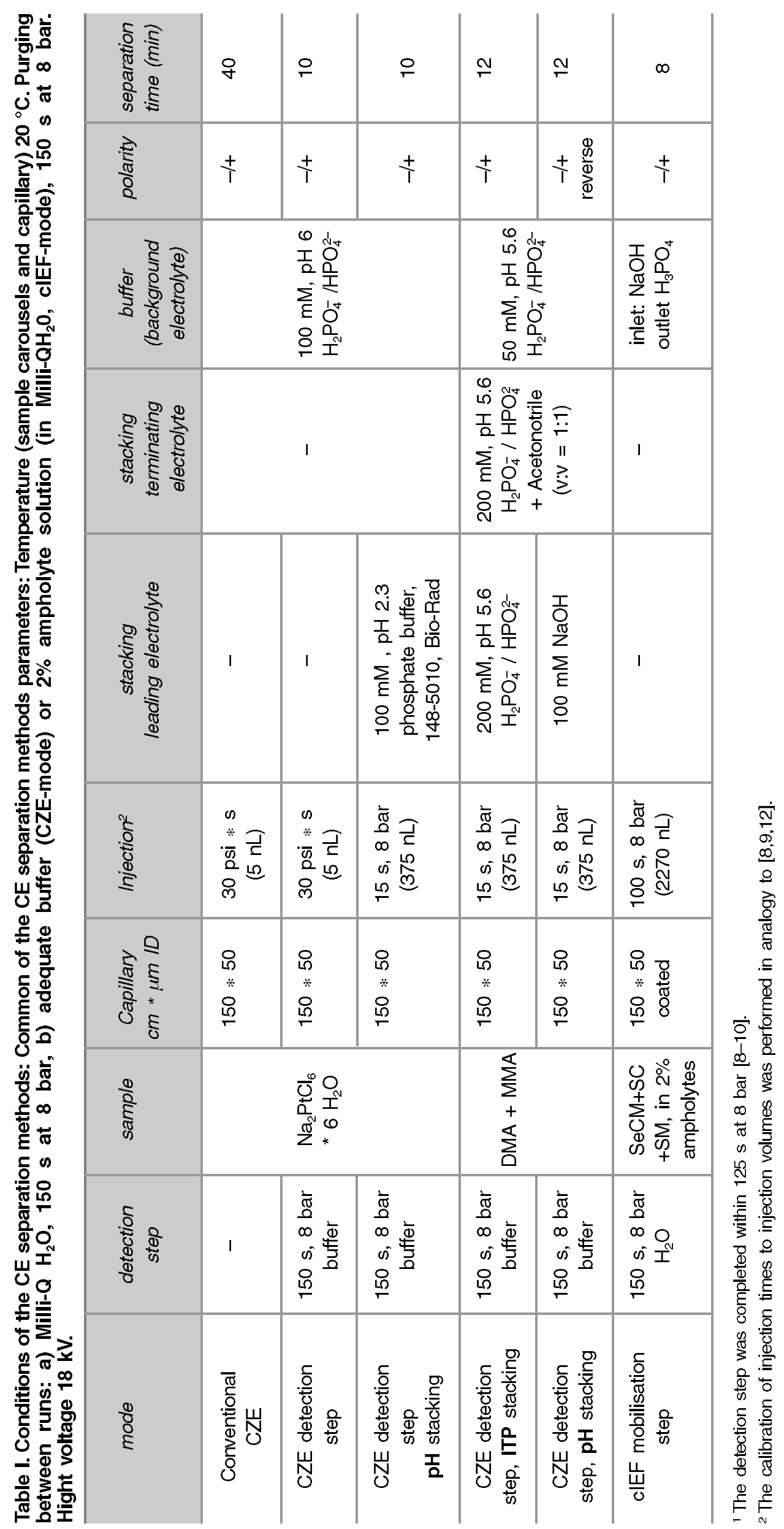



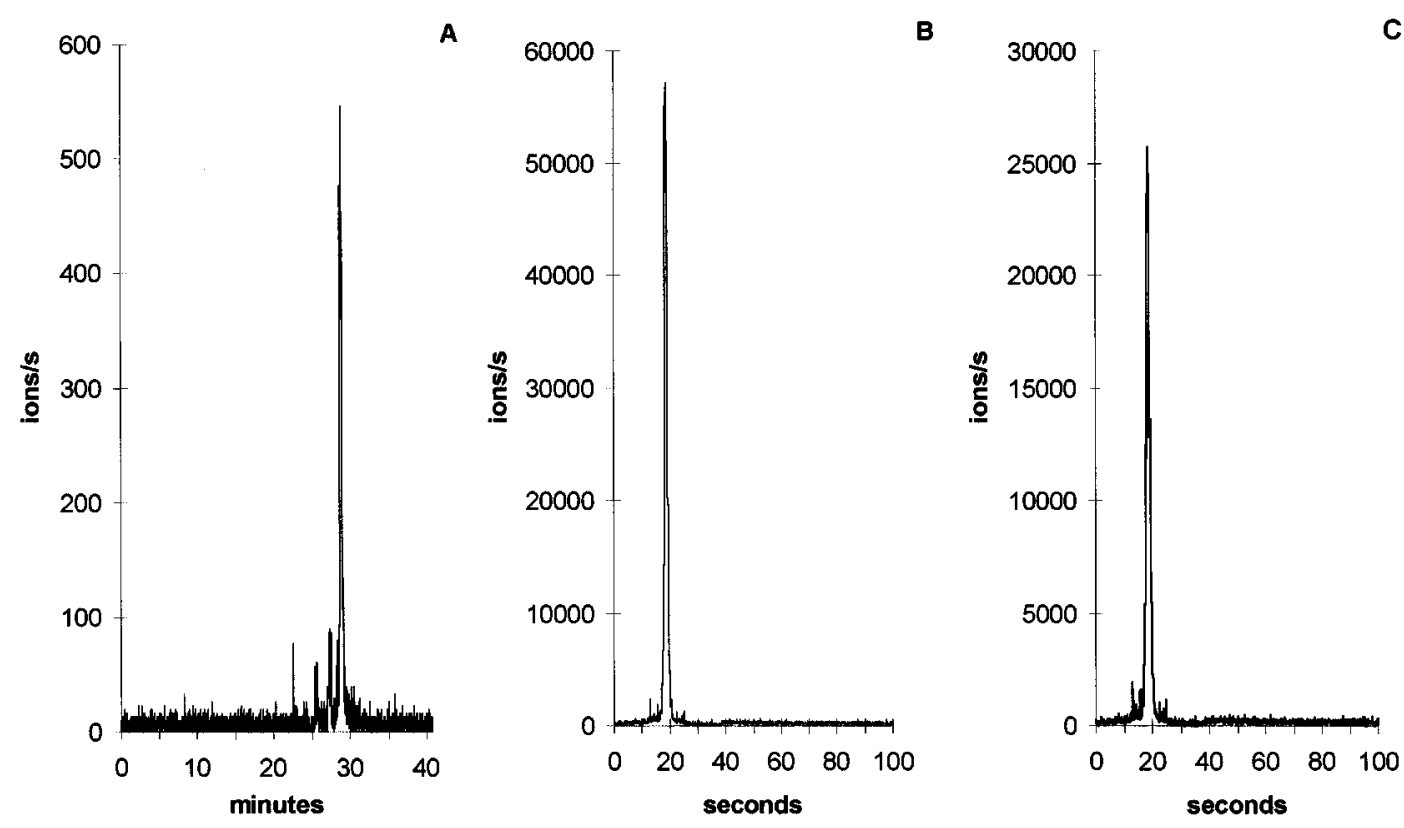

Figure 1. Electropherograms of $\mathrm{Na}_{2} \mathrm{PtCl}_{6} \times 6 \mathrm{H}_{2} \mathrm{O}$ are shown. A) $100 \mathrm{mg} / \mathrm{L}$, conventional operation, B) $100 \mathrm{mg} / \mathrm{L}$, division of separation and detection, C) $100 \mu \mathrm{g} / \mathrm{L}$ after ITP stacking, division of separation and detection.
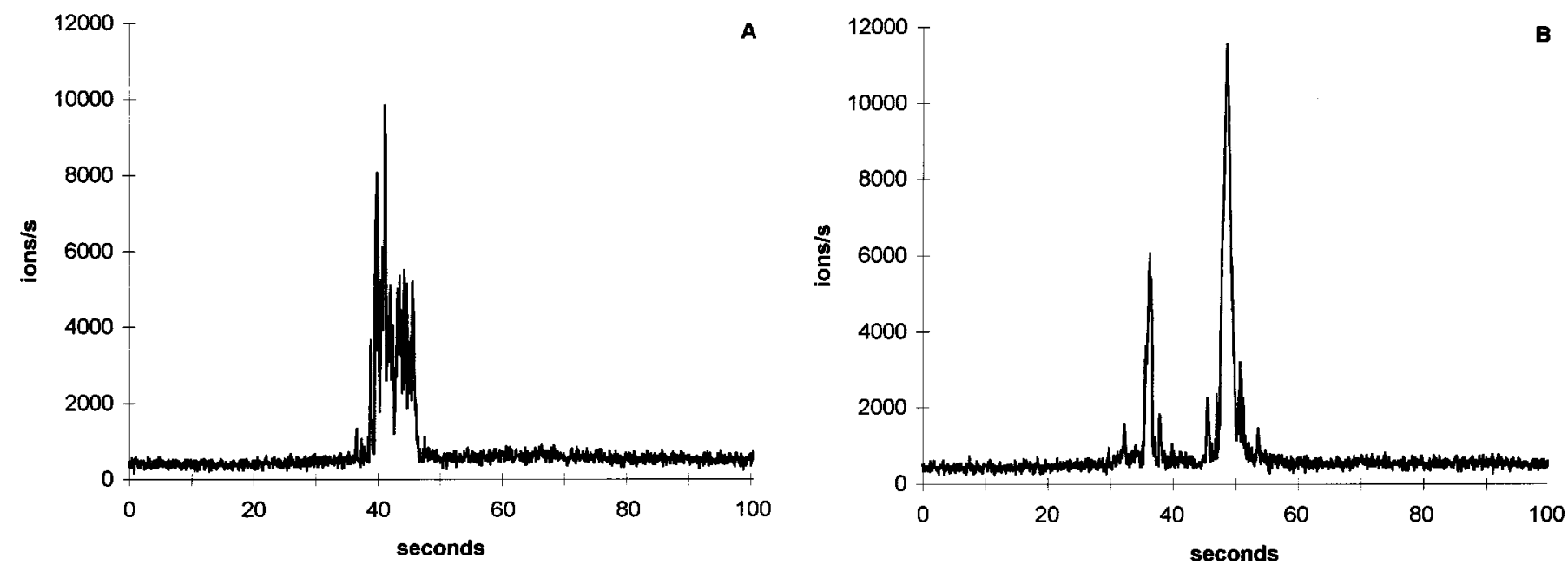

Figure 2. Improvement of separation of DMA and MMA. A) Operation with ITP stacking. Both As-species show the same movement direction and are hardly separated. B) Operation with pH stacking in the middle of the capillary. DA shows partly reversed movement. The As-species are separated clearly.

\section{Improvement of separation}

The improvement of separation is demonstrated with two arsenic species, monomethylarsonic acid (MMA) and dimethylarsonic acid (DMA). Both were just separated when using low sample volumes and long separation times. But when increasing the sample volume for improving concentration DL and applying a 12 min separation the signals were distorted and peaks were not resolved (Fig. 2A). However, figure 2B shows a good separation of the two As-species. Here, the sample was positionated in the middle of the capillary between the leading electrolyte and the terminating electrolyte. The alcaline (high) $\mathrm{pH}$ of the leading electrolyte obviously resulted in an initial backward movement. This was checked by positioning the sample (conventionally) at the beginning of the capillary and the terminating electrolyte was only in the inlet buffer vial.

When performing this experiment MMA was not monitored during separation or detection step. But analysing the inlet buffer directly after the run by ETV-ICP-MS, arsenic of MMA was seen there, proving the backward movement of this compound. Therefore, positioning such a sample in the middle of a long capillary and performing, both, backward movement and forward movement of the different species can help to improve separation. This is clearly demonstrated by comparing Figures $2 \mathrm{~A}$ and $2 \mathrm{~B}$. 


\section{Application of clEF}

Figures 3A and 3B show the mobilisation step of selenomethionine (SM), selenocystamine (SCM) and selenocysteine (SC) after 8 min focusing. Focusing was ended when the current dropped from ca. $17 \mathrm{~mA}$ to zero within $60 \mathrm{~s}$. This was the clear indication that the optimal focusing was achieved and mobilisation can start. The electropherograms show three major peaks at $21 \mathrm{~s} 34 \mathrm{~s}$ and $43 \mathrm{~s}$ at $\mathrm{m} / z=77$ and $m / z=78$, corresponding to the three Se-species. Also, several minor signals are monitored at both masses, each of them not paralleled in the other mass or showing not the (correct) natural isotope ratio. This fact identify that signals not to be authentic Se-signals, but polyatomic interferences. The three major peaks, however, correspond well with the natural isotope ratio of ${ }^{78} \mathrm{Se}$ and ${ }^{77} \mathrm{Se}$.

The three Se-species are clearly separated from each other, showing narrow peak width at half-height (ca. $0.5 \mathrm{~s}$ ) and high resolution. The concentration DL was calculated at $10 \mu \mathrm{g} / \mathrm{L}$.
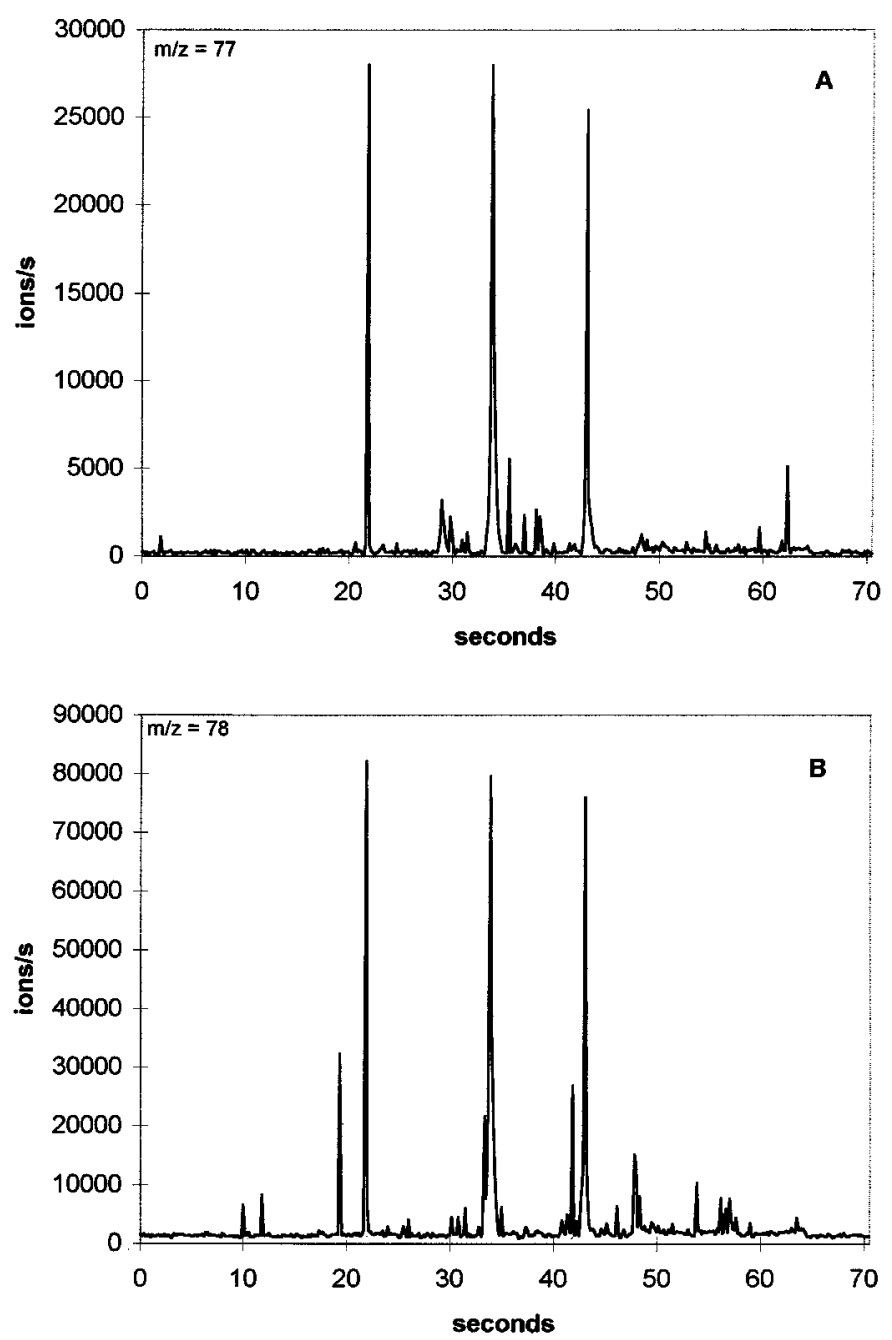

Figure 3. Mobilisation step of SM, SeCM and SC after $8 \mathrm{~min}$ clEF-focusing (pH $2-10)$. A) $m / z=77$, little polyatomic interferences, $B$ ) $m / z=78$, more polyatomic interferences.

\section{Discussion}

\section{Time reduction, improvement of nebulisation and concentration detection limit}

According to [12] species like $\mathrm{Se}(\mathrm{VI})$ or $\mathrm{Na}_{2} \mathrm{PtCl}_{6} \times 6 \mathrm{H}_{2} \mathrm{O}$ (also according this paper) reach the ICP-MS within ca. $30-35$ min when using conventional operation. The time reduction in the method developed is around $15-20 \mathrm{~min}$ for these species. But slower migrating species take up to $120 \mathrm{~min}$ or longer for being monitored conventionally [12]. In such a case, time reduction would be considerable. The nebulisation efficiency is also improved, as demonstrated by the comparison of figures $1 \mathrm{~A}$ and $1 \mathrm{~B}$. This has its origin in several effects:

(i) Charged molecules try to migrate to the outlet electrode. In case of a low retiring velocity during conventional operation they are leaving the CE-capillary and (partly) are moving immediately to the outlet electrode, without being nebulised. Only a part of the species is nebulised and transported into ICP-MS [8]. This entails a loss of sensitivity. The decreased sensitivity is avoided by increasing the retiring velocity (as shown for the pressure driven detection step: $1.5 \mu \mathrm{L} / \mathrm{min})$. During the detection step the velocity of species into the nebulisation gas stream is increased, resulting in an improvement of nebulisation and mass transport into ICP-MS

(ii) Previous experiments showed that the optimised flow for nebulisation was $1-2 \mu \mathrm{L} / \mathrm{min}$ when a laboratory made interface was employed [8]. This flow rate can be achieved by increasing the sheath flow, but no satisfactory results can be obtained in this way. The reason is a considerable dilution of the separated species with sheath buffer, leading to a loss in concentration DL.

(iii) Peak area is a constant value for a given detector sensitivity/instrumentation and analyte concentration. It is further a function of peak height and the time, the analyte passes the detector. Therefore, peak height is increasing due to a constant peak area, when reducing this time by accelerating the analyte [8].

A fiurther improvement of concentration DL is gained by sample stacking. Actually, this is not only typical for our mode of operation. But large capillary length enables an increased sample volume reaching up to $400 \mathrm{~nL}$ additional to an nearly equal amount of stacking buffer without a loss in resolution: the peak width at half-height of $1.85 \mathrm{~s}$ after injecting $375 \mathrm{~nL}$ (+ sample stacking; Fig. 1C) is only insignificantly larger than that one of $1.5 \mathrm{~s}$ after injection of $4 \mathrm{~nL}$ (without sample stacking; Fig. 1B). It was shown that the sample/stacking buffer "sandwich" in these experiments reached $30 \mathrm{~cm}$ of capillary length. When using "normal" capillaries (length typically $30-60 \mathrm{~cm}$ ) there is (nearly) no remainig space for stacking and CZE separation.

\section{Improvement of separation}

The $\mathrm{pH}$ stacking provides an alcaline leading electrolyte. The fast $\mathrm{OH}^{-}$ions migrate very fast to the anode, resulting in a successive decrease of $\mathrm{pH}$ in the capillary. At $\mathrm{pH} 8.2$ the pKa of MMA is passed and MMA changes its mobility. It takes some time during $\mathrm{pH}$ decrease until $\mathrm{pKa}$ of DMA 
is reached at $\mathrm{pH}$ 6.2. This time difference is used for separation as MMA shows partly opposite movement under the applied conditions. For monitoring these species the positioning of the sample in the middle of the capillary was necessary. Otherwise, the species (MMA) were found in the inlet buffer vial.

Another way for detecting, both, backward and forward moving compounds (or cations and anions) in one run is the application of an EOF. But in case of fast ions the EOF must be even faster and thus may interfere the separation [13]. Besides, an EOF is not available in acidic conditions, which is a principle limitation. The described procedure is independent on an EOF (no limitation) and a possible alteration of the separation by EOF also had not to be concerned with.

\section{Application of cIEF}

This technique is only applicable using the detection step for mobilisation. Typically its separation potential is superior to CZE and also the sample intake is higher. This improves the concentration DL. Although, Se is an element where only minor abundant isotopes $(\mathrm{m} / \mathrm{z}=77: \cong 8 \%$ of natural isotope amount of Se, $m / z=78$ : $\cong 24 \%$ of natural isotope amount of Se) can be monitored by ICP-MS, the concentration DL was improved by a factor of $3-5$ for organic Se-species compared to CZE [18].

\section{Possible problems}

In conventional $\mathrm{CE}$ molecule plugs show sharp borders, corresponding to separation principles of CE [16]. But the application of a pressure driven flow effects an undesirable laminar stream, changing plug borders to a more parabolic shape. Peak broadening and a loss of resolution usually is the consequence $[16,17]$.

But when looking at the electropherograms in former investigations [19] the resolution is excellent. There, six Ptspecies from a soil extract were baseline separated within $25 \mathrm{~s}$ during the detection step (three of these species were separated within $8 \mathrm{~s}$, each having a peakwidth at half-height of $1.2 \mathrm{~s}$ ). Probably the loss in resolution is negligible compared to the total (high) resolution. Furthermore, new software releases of commercial CE systems allow the applica- tion of high voltage during pressure for minimizing distortions of separation.

\section{References}

1. Harms, J.; Schwedt, G. Fresenius' J. Anal. Chem. 1994, 350, 93-100.

2. Weber, G. Fresenius' J. Anal. Chem. 1993, 346, 639-642.

3. Gardiner, P. E.; Ottaway, J. M.; Fell, G.; Burns, R. R. Anal.Chim. Acta 1981, 124, 281-294.

4. Gardiner, P. E.; Rösick, E.; Rösick, U.; Brätter, P.; Kynast, G. Clin. Chim. Acta 1982, 120, 103-117.

5. Lönnerdal, B.; Hoffmann, B. Biol. Trace Elem. Res. 1981, 3, 301-308.

6. Olesik, J. W.; Kinzer, J. A.; Olesik, S. V. Anal. Chem. 1995, 67(1), 1-12.

7. Liu, Y.; Lopez-Avila, V.; Zhu, J. J.; Wiederin, D. R.; Beckert, W. F. Anal. Chem. 1995, 67, 2020-2025.

8. Michalke, B.; Schramel, P. Fresenius' J. Anal. Chem. 1997, 357, 594-599.

9. Michalke, B.; Lustig, S.; Schramel, P. Electrophoresis 1997, 18, 196-201.

10. Michalke B, Schramel P, Electrophoresis, 1998, 19, 270-275.

11. Lewis, D.; Taylor, K. A.; Crews, H. M.; Sharp, B. L. $5^{\text {th }}$ International Symposium on Hyphenated Techniques in Chromatography and Hyphenated Chromatographic Analyzers, Bruges (Belgium), 11.-13. February 1998.

12. Michalke, B.; Schramel, P. J. Chromatography A 1996, 750, 51-62.

13. Schlegel, D.; Mattusch, J.; Wennrich, R. $26^{\text {th }}$ International Symposium on Environmental Analytical Chemistry, Wien (Austria), 9.-12. April 1996.

14. Formeln Physik Chemie Mathematik, Buch und Zeit Verlag $\mathrm{GmbH}$, Köln, 1980.

15. Chen, S. M.; Wiktorowicz, J. E. Anal. Biochem. 1992, 206, 8490.

16. Foret, F.; Müller, O.; Thorne, J.; Götzinger, W.; Karger, B. L. J. Chromatography A 1995, 716, 157-166.

17. Kilar, F. $2^{\text {nd }}$ Göttinger Capillary Elektrophoresis Symposium, Göttingen (Germany) 12.-13. October 1994.

18. Michalke, B.; Schramel, P. J. Chromatography A 1998 (in press).

19. Lustig, S.; Michalke, B.; Beck,W; Schramel, P. Fresenius' J. Anal. Chem. 1998, 360, 18-25. 